\title{
Gut Microbiota Resilience: Definition, Link to Health and Strategies for Intervention
}

\author{
Shaillay Kumar Dogra ${ }^{1}$, Joel Doré2 and Sami Damak ${ }^{1 *}$ \\ ${ }^{1}$ Nestlé Research, Société des Produits Nestlé SA, Lausanne, Switzerland, ${ }^{2}$ Université Paris-Saclay, Institut national \\ de recherche pour l'agriculture, I'alimentation et l'environnement, MetaGenoPolis, AgroParisTech, Microbiologie \\ de l'Alimentation au Service de la Santé, Jouy-en-Josas, France
}

\section{OPEN ACCESS}

Edited by: Carlotta De Filippo, National Research Council (CNR), Italy

Reviewed by:

Maria Jose Gosalbes, Centre for Biomedical Network

Research (CIBER), Spain Alinne Castro,

Dom Bosco Catholic University, Brazil

*Correspondence: Sami Damak sami.damak@rdls.nestle.com

Specialty section:

This article was submitted to Microbial Symbioses, a section of the journal Frontiers in Microbiology

Received: 15 June 2020

Accepted: 24 August 2020 Published: 15 September 2020

Citation:

Dogra SK, Doré J and Damak S (2020) Gut Microbiota Resilience: Definition, Link to Health and Strategies for Intervention.

Front. Microbiol. 11:572921. doi: 10.3389/fmicb.2020.572921
The gut microbiota is a new frontier in health and disease. Not only many diseases are associated with perturbed microbiota, but an increasing number of studies point to a cause-effect relationship. Defining a healthy microbiota is not possible at the current state of our knowledge mostly because of high interindividual variability. A resilient microbiota could be used as surrogate for healthy microbiota. In addition, the gut microbiota is an "organ" with frontline exposure to environmental changes and insults. During the lifetime of an individual, it is exposed to challenges such as unhealthy diet, medications and infections. Impaired ability to bounce back to the pre-challenge baseline may lead to dysbiosis. It is therefore legitimate to postulate that maintaining a resilient microbiota may be important for health. Here we review the concept of resilience, what is known about the characteristics of a resilient microbiota, and how to assess microbiota resilience experimentally using a model of high fat diet challenge in humans. Interventions to maintain microbiota resilience can be guided by the knowledge of what microbial species or functions are perturbed by challenges, and designed to replace diminished species with probiotics, when available, or boost them with prebiotics. Fibers with multiple structures and composition can also be used to increase microbiota diversity, a characteristic of the microbiota that may be associated with resilience. We finally discuss some open questions and knowledge gaps.

Keywords: microbiome, resilience, probiotic, fiber, health

\section{INTRODUCTION}

The human gut harbors trillions of microbes, essentially bacteria, counting more than 3000 different bacterial species with each individual harboring 200 to 300 species (Qin et al., 2010). This microbial ecosystem, the microbiota, plays a crucial role in human physiology and health (Flint et al., 2012; Gentile and Weir, 2018). Research on gut microbiota has historically focused on associations with diseases such as Irritable Bowel Syndrome (Menees and Chey, 2018), Inflammatory Bowel Disease (Ott et al., 2004; Manichanh et al., 2006), allergy (Ho and Bunyavanich, 2018), diabetes (Sharma and Tripathi, 2018), cancer (Baba et al., 2017; Dahmus et al., 2018), asthma (Fujimura and Lynch, 2015), and obesity (Furet et al., 2010). A few studies have gone beyond association and suggested causative effect of the microbiota on certain diseases, based either on fecal transplants in humans (Vrieze et al., 2012; Philips et al., 2017; Palleja et al., 2018; Chen et al., 2019; Costello et al., 2019; Kang et al., 2019) or on human microbiota transfer to germ-free mice (Le Roy et al., 2013; Duca et al., 2014; Peng et al., 2014; Kelly et al., 2016; Llopis et al., 2016; Schaubeck et al., 2016; 
Routy et al., 2018; Sharon et al., 2019; Kim et al., 2020). The adult gut microbiota is fairly stable while constantly being influenced by the host and by multiple external factors. Triggered by particularly strong stressors the gut microbiota may be critically modified and this could impact individual's health.

\section{HIGH VARIATION OF MICROBIOTA COMPOSITION AND HOW TO DEFINE A HEALTHY MICROBIOTA}

Defining a healthy microbiota is very important for being able to prevent or correct dysbiosis and minimize its impact on health. However, the composition of the microbiota is very diverse and highly variable, depending on diet (Orwin and Wardle, 2004; Wu et al., 2011; Johnson et al., 2019), geographic location (Gupta et al., 2017; He et al., 2018), ethnicity (Deschasaux et al., 2018), level of exercise (O'Sullivan et al., 2015; Cook et al., 2016; Mach and Fuster-Botella, 2017; Barton et al., 2018), medication use including but not limited to antibiotics (Zaura et al., 2015; Imhann et al., 2016; Jackson et al., 2018), and genetics (Goodrich et al., 2014). In addition to these intrinsic and extrinsic modulators, a large part of the microbiota variation between individuals cannot be explained by any specific factor (Walker et al., 2011; Wu et al., 2011).

The high degree of variability makes it difficult to define a normal or healthy microbiota (Mardinoglu et al., 2018; McBurney et al., 2019). Yet some parameters such as increased diversity, gene richness, or proportion of butyrate producers are often considered as features of a healthy gut microbiota (reviewed in Lemon et al., 2012; Lloyd-Price et al., 2016). The impact on host parameters such as the gut barrier function (Natividad and Verdu, 2013) and immunity (Ye et al., 2018) can also be taken into consideration when determining if the gut microbiota is healthy or dysbiotic.

Alternatively, in case of gut microbial ecosystems, resilience can be used as a surrogate marker of a healthy ecosystem (Allison and Martiny, 2008; Banning and Murphy, 2008; Lozupone et al., 2012; Greenhalgh et al., 2016; Sommer et al., 2017; Fraccascia et al., 2018). Resilience is the property of an ecosystem to resist changes under stress or to quickly and fully recover from the perturbations (Ingrisch and Bahn, 2018, Box 1).

\section{MICROBIOTA RESILIENCE}

Linked together with functional and life-sustaining interdependencies, bacteria inhabiting the human gut are structured as a complex ecosystem with multiple cross-talk (Costello et al., 2012; Coyte et al., 2015; Gilbert and Lynch, 2019). Such a gut microbial ecosystem evolves and establishes itself during early life from neonate to infants to toddlers (Backhed et al., 2015; Dogra et al., 2015; Stewart et al., 2018) and stays mostly stable in adult life (Mehta et al., 2018). External stress factors such as extreme dietary changes (David et al., 2014; Mardinoglu et al., 2018), infections (Hsiao et al., 2014), antibiotics usage (Jernberg et al., 2007; Dethlefsen and Relman, 2011; Willing et al., 2011;
Palleja et al., 2018) or other medications, including members of all therapeutic classes (Le Bastard et al., 2018; Maier et al., 2018) perturb this ecosystem. Subsequently, this gut microbial ecosystem may or may not return to its original state. This is conceptually illustrated in Figure 1, inspired by Folke et al. (2004). A resilient microbiota will return to its original state of equilibrium after being subjected to a perturbation, whereas a non-resilient microbiota will shift to an altered new state.

It can be argued that the microbiota of a healthy individual with the ability to return quickly and fully to baseline after a challenge is a healthy microbiota because this resilience may prevent the establishment of a new equilibrium and drift toward dysbiosis, with negative impact on the individual's health (Sommer et al., 2017).

The concept of gut microbiota resilience has been discussed in several reviews and perspective articles (Relman, 2012; LloydPrice et al., 2016; Sommer et al., 2017). As recently proposed, a model combining challenge tests and biomarkers that can inform on the dynamics of microbiota recovery allows to document resilience as an indicator of health (Taroncher-Oldenburg et al., 2018). However, these proposals remain theoretical and to our knowledge, experimental validation of models for quantification of resilience has not been reported.

\section{WHAT ARE THE CHARACTERISTICS OF A RESILIENT MICROBIOTA?}

Diversity may be a positive contributor to resilience. Tap et al. (2015) found that the human gut microbiota richness increases its stability when challenged by increased dietary fiber intake. Another human study showed that a weaker antibiotic-induced perturbation of the microbiota is linked to higher pre-challenge microbiota diversity (Raymond et al., 2016).

Host immune status may also affect microbiota resilience. Two studies have shown that genetic ablation of the bacterial sensor nod 2 in mice leads to impaired recovery of the microbiota from antibiotic perturbation (Goethel et al., 2019; Anderson et al., 2020). However, a third study using aroA-deficient Salmonella as challenge did not find any effect of the nod proteins on microbiota resilience (Robertson et al., 2016). Nod is an intracellular sensor of immune signals especially sensitive to specific peptidoglycan structures, hence reactivity may depend on the pathogen used in the challenge. These elegant basic research studies suggest that stability of host-microbiota symbiosis may be another important metric to assess resilience.

\section{BOX 1 | Definitions}

Baseline is the state of an ecosystem before perturbation.

Impact is the change in the ecosystem because of a stressor.

Resilience is the property of an ecosystem to maintain its state and recover from perturbations. The capacity of the system to persist during the impact (resistance) and to return to baseline after the impact of disturbance (recovery) determines overall resilience. Conceptually, this has been illustrated in

Figure 2 below. 


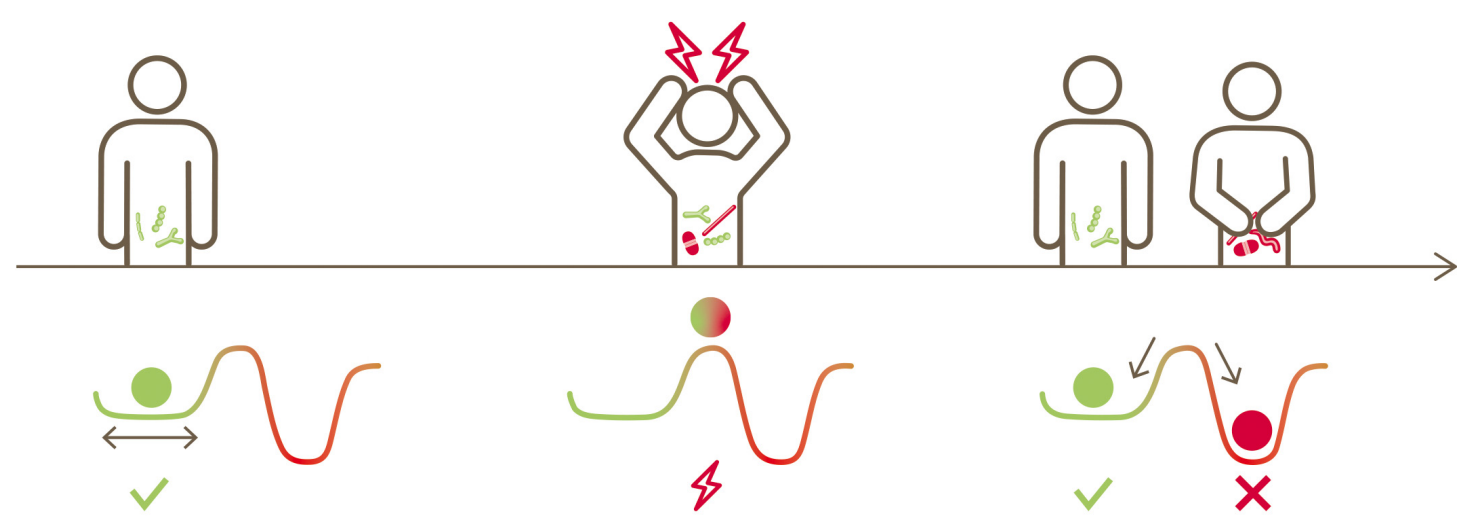

FIGURE 1 | Conceptual illustration depicting the native state of an ecosystem, its perturbation, the possibility of returning to its original state or transitioning to a new state (alternative stable state) (inspired by Folke et al., 2004).

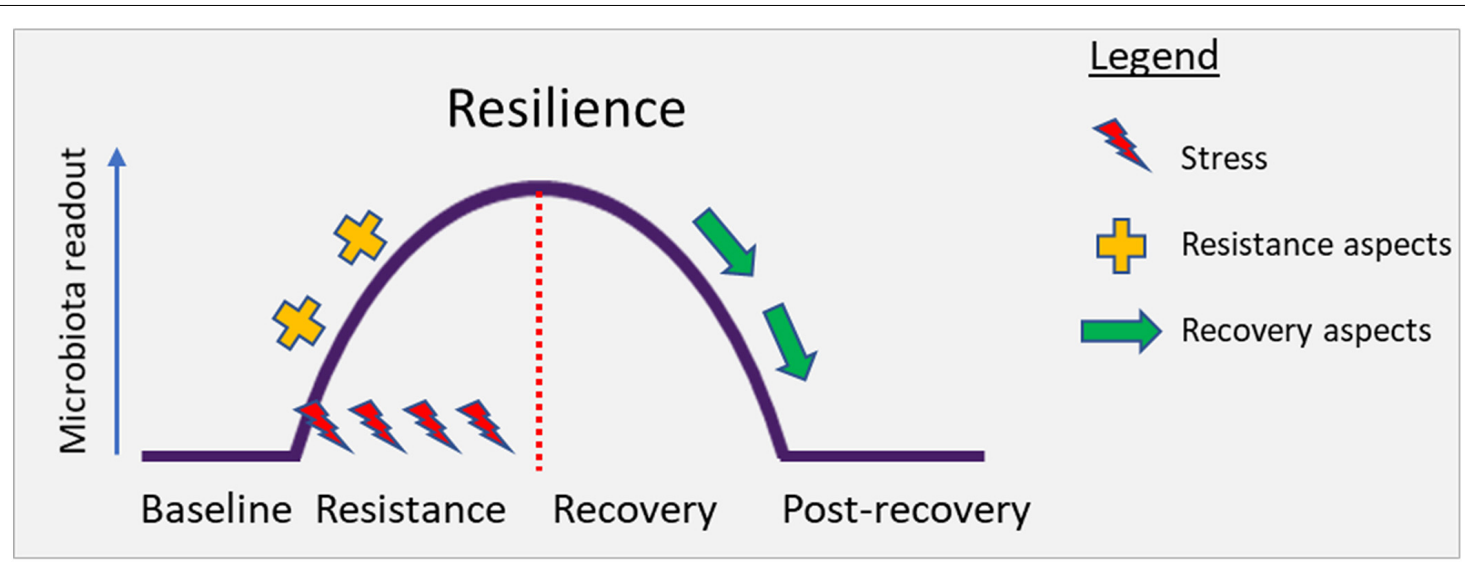

FIGURE 2 | Conceptual illustration depicting the perturbation of the gut microbiota under stress. Resilience describes the capacity of an ecosystem to maintain its state or recover from disturbances. Resilience is determined by the capacity to reduce the impact (resistance) and to recover from the impact of disturbance (recovery) (Ingrisch and Bahn, 2018).

A few authors have attempted to model stability and recovery of the gut microbial ecosystem. Shaw et al. (2019) used stability landscape frameworks to model recovery of the gut microbiota after treatment with antibiotics. Certain other methods, such as increased auto-correlation and variance of the variables in the 'dominant' groups, can be early indicators of critical transitions to alternative stable states (Scheffer et al., 2009; Liu et al., 2015). One study focused on defining the 'tipping elements' in the microbiota by looking into the distribution of specific microbiota species and found bi-modal distributions that associate with host parameters. Some of the bacteria were either hardly present or quite abundant, depending on certain host factors, and may be those associated with the shift to an altered state (Lahti et al., 2014).

\section{ASSESSING MICROBIOTA RESILIENCE EXPERIMENTALLY}

There are a few formulae for resilience described in the literature (Orwin and Wardle, 2004; Banning and Murphy, 2008; Fraccascia et al., 2018; Lourenco et al., 2018; Ye et al., 2018). Most of these were reviewed recently and categorized into a conceptual framework (Bahn and Ingrisch, 2018; Ingrisch and Bahn, 2018; Yeung and Richardson, 2018). Briefly, these formulae can be classified into three types of metrics: one describes change in relation to the baseline from the pre-disturbance state, thereby assessing to which extent the system gets perturbed; the other two metrics look into recovery after disturbance. This recovery can either be formulated as relative to baseline or relative to magnitude of perturbation. The former emphasizes how well the system has returned to its pre-perturbation state. The latter emphasizes how much it has recovered from the impact of perturbation.

In order to assess microbiota resilience experimentally, one must apply a challenge to the microbiota and measure some or all the above-mentioned parameters. A few studies have used an antibiotic or dietary challenge to perturb the microbiota and assess the impact of various interventions on this perturbation (Engelbrektson et al., 2009; Odamaki et al., 2016; RodriguezMorato et al., 2018). 
The David et al. (2014) study provides a good model to study microbiota resilience in human trials. The challenge it used is diet of animal origin (e.g., meat, cheese), made of $70 \%$ fat, $0 \%$ carbohydrates, and $30 \%$ protein. Microbiota composition changed significantly as early as after 2 days of dietary intervention and returned to baseline 6 days after the end of the diet challenge. The advantage of using a dietary challenge is that it can be standardized, and does not raise ethical issues, as using antibiotics in healthy subjects might do.

A resilience index can be computed by using parameters that are known to be modified by stress such as microbiota composition, function and/or metabolites. The resilience index can be used to give a quantitative measure of how much the microbiota has deviated and how quickly and fully it has recovered, thereby quantifying resilience. Such a resilience index provides a measure which can possibly be used to compare different stressors across studies.

Ideally, one should be able to assess the microbiota resilience of an individual without applying a stressor. Machine learning with comprehensive and big data may help design algorithms based on microbiota and host parameters that can be used to predict microbiota resilience. One such parameter is $\alpha$-diversity. This is supported by a human study showing that a weaker antibiotic-induced perturbation of the microbiota is linked to higher pre-challenge microbiota $\alpha$-diversity (Raymond et al., 2016). A study using microbiota transfer into germfree mice showed that the magnitude of antibiotic induced perturbation was donor dependent (Lavelle et al., 2019), giving ground to the hypothesis that it could be possible to predict resilience by analyzing the baseline microbiota in the absence of a challenge.

\section{WHAT IS THE RELATIONSHIP OF GUT MICROBIOTA RESILIENCE TO HEALTH?}

As discussed above, throughout the lifetime the gut microbiota is subjected to repeated and diverse challenges, including unhealthy diet, medications, alcohol, intense exercise, and pathogens, to just name some. Decreased ability to resist these challenges or to return quickly and fully to the prechallenge state may lead to a new equilibrium and dysbiosis, which may contribute to the development of chronic noncommunicable diseases (CNCDs). The diminished ability of the microbiota to return to baseline and establishment of a new equilibrium has been observed in human subjects following treatment with antibiotics (Dethlefsen and Relman, 2011). Thus, intervening to maintain the microbiota in a resilient state may constitute a measure to delay or prevent the development of microbiota related CNCDs. It is noteworthy that the microbiota constitutes an easily accessible "organ" for intervention, although how to stably modulate the microbiota may be less evident.

This theory of a link between microbiota resilience and health is attractive but there are very few data to support it. In a human study, Mondot et al. (2016) defined microbiota robustness as high species richness and high inter-OTU (operational taxonomic unit) correlation. They found that microbiota robustness was positively associated with Crohn disease remission after ileocolic resection (Mondot et al., 2016).

\section{WHAT ARE THE POSSIBLE MEANS OF INTERVENTION?}

\section{Fibers}

Knowing the characteristics of a resilient microbiota will help design the interventions aimed at increasing resilience. While this knowledge is currently incomplete, it is already known that diversity may be a contributing parameter (Tap et al., 2015; Raymond et al., 2016) and dietary fibers may be a way of increasing microbiota diversity. Dietary fibers are carbohydrate polymers that are not digested nor absorbed in the upper gastrointestinal tract and reach the colon where they are subjected to bacterial fermentation. Many studies have shown that fibers impact the composition and function of the microbiota, especially the production of short chain fatty acids (for review see, Holscher, 2017). Studies of humans in different geographical locations showed that greater dietary fiber intake is associated with increased gut microbiota diversity (Rampelli et al., 2015) and human intervention studies have revealed that dietary fiber and whole grain intake increase the diversity of the gut microbiota (Martinez et al., 2013; Tap et al., 2015). Microbiota diversity could in principle also be increased by a complex mix of dietary fibers providing a wide range of structures and monosaccharide units as indicated by at least one mouse study (Cheng et al., 2017). A study in mice has shown that fibers have a direct effect on improving microbiota resilience. In this study, the microbiota of mice fed with a fiber enriched diet and challenged with antibiotic and Clostridioides (formerly Clostridium) difficile returned to pre-challenge composition whereas the microbiota of mice fed a low fiber diet and challenged in the same way did not (Hryckowian et al., 2018).

\section{Probiotics and Dietary Interventions Boosting Certain Microbial Species}

Published data on human microbiota submitted to various challenges (Hsiao et al., 2014; Mardinoglu et al., 2018; Palleja et al., 2018) showed a number of species that are commonly perturbed. Those may be fragile species that could be boosted or replenished by nutritional intervention. For example, Bifidobacterium adolescentis is the most diminished species with high fat diet (Mardinoglu et al., 2018). In addition, this species is highly reduced and slow to recover following a challenge with antibiotics (Palleja et al., 2018). It is therefore reasonable to put forward and (i) test the hypothesis that providing $B$. adolescentis as component of a probiotic blend may improve the resilience of the microbiota and (ii) exclude the possibility that $B$. adolescentis is just a bioindicator. Other Bifidobacterium and Lactobacillus species that exist as probiotics such as B. longum, B. bifidum, B. angulatum, and L. casei are 
also altered by challenges to the microbiota (Mardinoglu et al., 2018; Palleja et al., 2018), although not as dramatically as $B$. adolescentis, and hence, could be part of a resilience blend nutritional solution. Not all challenge-diminished species exist as probiotics, but ingredients known to boost those species may also be used to prevent their decline during and after a challenge. For example, inulin favors the growth of $B$. adolescentis, and of Faecalibacterium prausnitzii (Ramirez-Farias et al., 2009). F. prausnitzii is a beneficial species that is diminished when the microbiota is challenged with antibiotics or high fat diet and that is associated with recovery from diarrhea (Hsiao et al., 2014; Mardinoglu et al., 2018; Palleja et al., 2018). Dietary interventions aimed at boosting certain species must be carefully dosed so that the targeted species does not become too prominent and negatively affect $\alpha$-diversity, which will be against the desired effect.

Two studies investigated the effect of probiotics on perturbations of the microbiota by antibiotics to determine if they would improve resilience. One study showed no effect of supplementation with Lactobacillus rhamnosus and Lactobacillus helveticus on microbiota perturbations induced by the antibiotics amoxicillin + clavulanic acid (MacPherson et al., 2018) whereas a second older study showed that a mix of probiotics (B. lactis $\mathrm{Bl}-04$, B. lactis $\mathrm{Bi}-07$, Lactobacillus acidophilus NCFM, Lactobacillus paracasei Lpc-37, and Bifidobacterium bifidum $\mathrm{Bb}-02)$, minimizes the amoxicillin + clavulanic acid-induced disruption of fecal microbiota (Engelbrektson et al., 2009). The probiotic group in this study, showed a strong trend for improved recovery by random fragment length polymorphism (RFLP) analysis, and additionally a significant increase in resistance by bacterial culture.

Beyond repairing the microbiota composition, it is also relevant to consider how to minimize the effects of a perturbed microbiota on the host. Dysbiosis often leads to inflammation (Blander et al., 2017) and increased gut permeability (reviewed in Jandhyala et al., 2015), which in turn negatively impacts the microbiota. To break this vicious circle, interventions with a mix of probiotics containing species with proven antiinflammatory properties such as Lactobacillus rhamnosus GG (Capurso, 2019) or shown to strengthen the gut barrier such as Lactobacillus plantarum (Karczewski et al., 2010) may be a good complementary approach to improving the microbiota by acting on the host physiology.

\section{OPEN QUESTIONS}

Many questions remain to be answered on what other parameters make a microbiota resilient. The role of keystone species must be determined and may be important. These taxa are believed to interact with a large number of other taxa and may help maintain the status of the microbiota (Fisher and Mehta, 2014). At this point the role played by keystone species in microbiota resilience has not been clarified, and only a few candidate keystone species [e.g., Ruminococcus bromii (Ze et al., 2012)] have been proposed.
How about rare species? Typically, taxa with abundance below a certain threshold are rightfully excluded from analyses because the difference between a very low figure and zero might be due to method sensitivity and not to a physiological difference. This approach could be revisited for studies related to resilience, as the disappearance of low abundance taxa may be more relevant than a large decrease of an abundant one. If a low abundance species providing an important function becomes extinct, this irreversible event could shift the microbiota to a different stable state which may be a step toward dysbiosis.

The question remains open on whether knowledge at the species level is sufficiently reliable for intervention. The benefits of probiotics are known to be strain dependent. Therefore, providing a particular strain to replace a diminished species may not work, if it is another strain with a different function that is perturbed. Further improvements of the analysis techniques are needed since the current shotgun metagenomic microbiota analysis technology allows discrimination at the species level, but rarely at the strain level.

The role in microbiota resilience of gut microbes belonging to other kingdoms remains to be elucidated. Most studies of the microbiota have focused on bacteria, but archaea, eukaryotes, and viruses are also present in the gut. A few studies have found associations between the composition of virome, mycome or archaeome and human diseases, including type 2 diabetes, inflammatory bowel disease, and obesity (Blais Lecours et al., 2014; Bhute et al., 2017; Borges et al., 2018; Ghavami et al., 2018; Ma et al., 2018; Maya-Lucas et al., 2019; Zuo et al., 2019). Interestingly one study showed reduced virome diversity before development of autoimmunity in children susceptible to type 1 diabetes (Zhao et al., 2017). Very little is known about the interactions between kingdoms of the commensal gut microbes. To our knowledge there are no studies that suggest a role of viruses, archaea and fungi in mammalian gut microbiota resilience. Yet, it is possible that other organisms beyond bacteria play a role in resilience, especially phages since they have the ability to infect and lyse specific bacteria, thereby possibly controlling their numbers.

Another important question is whether the resilience of the gut microbiota is stress specific or is the degree of resilience similar regardless of the nature of the stress. At present this question has not been addressed experimentally.

\section{CLOSING REMARKS}

We summarized here the many challenges for understanding what makes a resilient microbiota and how to devise a strategy for an effective nutrition intervention to improve resilience. Improving microbiota resilience may have important beneficial effects on health. The current knowledge although incomplete, includes enough information to justify and guide the design of human trials of first-generation solutions. As we learn more about the mechanisms of microbiota resilience, future new findings will be used to design the composition of nextgeneration interventions. 


\section{AUTHOR CONTRIBUTIONS}

All authors contributed to writing the manuscript, reviewed and approved its final version.

\section{FUNDING}

Funding was from Société des Produits Nestlé internal sources and by funding to JD from the European Research Council

\section{REFERENCES}

Allison, S. D., and Martiny, J. B. (2008). Colloquium paper: resistance, resilience, and redundancy in microbial communities. Proc. Natl. Acad. Sci. U.S.A. 105(Suppl. 1), 11512-11519. doi: 10.1073/pnas.0801925105

Anderson, J. M., Lipinski, S., Sommer, F., Pan, W. H., Boulard, O., Rehman, A., et al. (2020). NOD2 influences trajectories of intestinal microbiota recovery after antibiotic perturbation. Cell. Mol. Gastroenterol. Hepatol. 10, 365-389.

Baba, Y., Iwatsuki, M., Yoshida, N., Watanabe, M., and Baba, H. (2017). Review of the gut microbiome and esophageal cancer: pathogenesis and potential clinical implications. Ann. Gastroenterol. Surg. 1, 99-104. doi: 10.1002/ags3.12014

Backhed, F., Roswall, J., Peng, Y., Feng, Q., Jia, H., Kovatcheva-Datchary, P., et al. (2015). Dynamics and stabilization of the human gut microbiome during the first year of life. Cell Host Microbe 17, 690-703. doi: 10.1016/j.chom.2015.04.004

Bahn, M., and Ingrisch, J. (2018). Accounting for complexity in resilience comparisons: a reply to Yeung and Richardson, and Further considerations. Trends Ecol. Evol. 33, 649-651. doi: 10.1016/j.tree.2018.06.006

Banning, N. C., and Murphy, D. V. (2008). Effect of heat-induced disturbance on microbial biomass and activity in forest soil and the relationship between disturbance effects and microbial community structure. Appl. Soil Ecol. 40, 109-119. doi: 10.1016/j.apsoil.2008.03.011

Barton, W., Penney, N. C., Cronin, O., Garcia-Perez, I., Molloy, M. G., Holmes, E., et al. (2018). The microbiome of professional athletes differs from that of more sedentary subjects in composition and particularly at the functional metabolic level. Gut 67, 625-633.

Bhute, S. S., Suryavanshi, M. V., Joshi, S. M., Yajnik, C. S., Shouche, Y. S., and Ghaskadbi, S. S. (2017). Gut microbial diversity assessment of Indian Type-2Diabetics Reveals Alterations in Eubacteria, Archaea, and Eukaryotes. Front. Microbiol. 8:214. doi: 10.3389/fmicb.2017.00214

Blais Lecours, P., Marsolais, D., Cormier, Y., Berberi, M., Hache, C., Bourdages, R., et al. (2014). Increased prevalence of Methanosphaera stadtmanae in inflammatory bowel diseases. PLoS One 9:e87734. doi: 10.1371/journal.pone. 0087734

Blander, J. M., Longman, R. S., Iliev, I. D., Sonnenberg, G. F., and Artis, D. (2017). Regulation of inflammation by microbiota interactions with the host. Nat. Immunol. 18, 851-860. doi: 10.1038/ni.3780

Borges, F. M., de Paula, T. O., Sarmiento, M. R. A., de Oliveira, M. G., Pereira, M. L. M., Toledo, I. V., et al. (2018). Fungal diversity of human gut microbiota among Eutrophic, overweight, and obese individuals based on aerobic culturedependent approach. Curr. Microbiol. 75, 726-735. doi: 10.1007/s00284-0181438-8

Capurso, L. (2019). Thirty years of Lactobacillus rhamnosus GG: a review. J. Clin. Gastroenterol. 53(Suppl. 1), S1-S41. doi: 10.1097/MCG.0000000000001170

Chen, D., Wu, J., Jin, D., Wang, B., and Cao, H. (2019). Fecal microbiota transplantation in cancer management: current status and perspectives. Int. J. Cancer 145, 2021-2031. doi: 10.1002/ijc.32003

Cheng, W., Lu, J., Li, B., Lin, W., Zhang, Z., Wei, X., et al. (2017). Effect of functional oligosaccharides and ordinary dietary fiber on intestinal microbiota diversity. Front. Microbiol. 8:1750. doi: 10.3389/fmicb.2017.01750

Cook, M. D., Allen, J. M., Pence, B. D., Wallig, M. A., Gaskins, H. R., White, B. A., et al. (2016). Exercise and gut immune function: evidence of alterations in colon immune cell homeostasis and microbiome characteristics with exercise training. Immunol. Cell Biol. 94, 158-163. doi: 10.1038/icb.2015.108

Costello, E. K., Stagaman, K., Dethlefsen, L., Bohannan, B. J., and Relman, D. A. (2012). The application of ecological theory toward an understanding
(ERC) under the European Union's Horizon 2020 Research and Innovation Programme (Grant Agreement ERC-2017-AdG No. 788191 - Homo.symbiosus). The funder was not involved in the writing of this article or the decision to submit it for publication.

\section{ACKNOWLEDGMENTS}

The authors thank Dr. Norbert Sprenger for critical review of the manuscript and constructive comments.

of the human microbiome. Science 336, 1255-1262. doi: 10.1126/science.122 4203

Costello, S. P., Conlon, M. A., and Andrews, J. M. (2019). Fecal microbiota transplantation for ulcerative colitis-reply. JAMA 321, 2240-2241. doi: 10.1001/ jama.2019.3950

Coyte, K. Z., Schluter, J., and Foster, K. R. (2015). The ecology of the microbiome: networks, competition, and stability. Science 350, 663-666. doi: 10.1126/ science.aad2602

Dahmus, J. D., Kotler, D. L., Kastenberg, D. M., and Kistler, C. A. (2018). The gut microbiome and colorectal cancer: a review of bacterial pathogenesis. J. Gastrointest. Oncol. 9, 769-777. doi: 10.21037/jgo.2018.04.07

David, L. A., Maurice, C. F., Carmody, R. N., Gootenberg, D. B., Button, J. E., Wolfe, B. E., et al. (2014). Diet rapidly and reproducibly alters the human gut microbiome. Nature 505, 559-563. doi: 10.1038/nature12820

Deschasaux, M., Bouter, K. E., Prodan, A., Levin, E., Groen, A. K., Herrema, H., et al. (2018). Depicting the composition of gut microbiota in a population with varied ethnic origins but shared geography. Nat. Med. 24, 1526-1531. doi: 10.1038/s41591-018-0160-1

Dethlefsen, L., and Relman, D. A. (2011). Incomplete recovery and individualized responses of the human distal gut microbiota to repeated antibiotic perturbation. Proc. Natl. Acad. Sci. U.S.A. 108(Suppl. 1), 4554-4561. doi: 10. 1073/pnas.1000087107

Dogra, S., Sakwinska, O., Soh, S. E., Ngom-Bru, C., Bruck, W. M., Berger, B., et al. (2015). Dynamics of infant gut microbiota are influenced by delivery mode and gestational duration and are associated with subsequent adiposity. mBio 6:e02419-14. doi: 10.1128/mBio.02419-14

Duca, F. A., Sakar, Y., Lepage, P., Devime, F., Langelier, B., Dore, J., et al. (2014). Replication of obesity and associated signaling pathways through transfer of microbiota from obese-prone rats. Diabetes 63, 1624-1636. doi: 10.2337/db131526

Engelbrektson, A., Korzenik, J. R., Pittler, A., Sanders, M. E., Klaenhammer, T. R., Leyer, G., et al. (2009). Probiotics to minimize the disruption of faecal microbiota in healthy subjects undergoing antibiotic therapy. J. Med. Microbiol. 58, 663-670. doi: 10.1099/jmm.0.47615-0

Fisher, C. K., and Mehta, P. (2014). Identifying keystone species in the human gut microbiome from metagenomic timeseries using sparse linear regression. PLoS One 9:e102451. doi: 10.1371/journal.pone.0102451

Flint, H. J., Scott, K. P., Louis, P., and Duncan, S. H. (2012). The role of the gut microbiota in nutrition and health. Nat. Rev. Gastroenterol. Hepatol. 9, 577-589. doi: $10.1038 /$ nrgastro.2012.156

Folke, C., Carpenter, S., Walker, B., Scheffer, M., Elmqvist, T., Gunderson, L., et al. (2004). Regime shifts, resilience, and biodiversity in ecosystem management. Annu. Rev. Ecol. Evol. Syst. 35, 557-581. doi: 10.1146/annurev.ecolsys.35. 021103.105711

Fraccascia, L., Giannoccaro, I., and Albino, V. (2018). Resilience of complex systems - state of the art and directions for future research. Complexity 2018:3421529. doi: 10.1155/2018/3421529

Fujimura, K. E., and Lynch, S. V. (2015). Microbiota in allergy and asthma and the emerging relationship with the gut microbiome. Cell Host Microbe 17, 592-602. doi: 10.1016/j.chom.2015.04.007

Furet, J. P., Kong, L. C., Tap, J., Poitou, C., Basdevant, A., Bouillot, J. L., et al. (2010). Differential adaptation of human gut microbiota to bariatric surgery-induced weight loss: links with metabolic and low-grade inflammation markers. Diabetes 59, 3049-3057. doi: 10.2337/db10-0253

Gentile, C. L., and Weir, T. L. (2018). The gut microbiota at the intersection of diet and human health. Science 362, 776-780. doi: 10.1126/science.aau5812 
Ghavami, S. B., Rostami, E., Sephay, A. A., Shahrokh, S., Balaii, H., Aghdaei, H. A., et al. (2018). Alterations of the human gut Methanobrevibacter smithii as a biomarker for inflammatory bowel diseases. Microb. Pathog. 117, 285-289. doi: 10.1016/j.micpath.2018.01.029

Gilbert, J. A., and Lynch, S. V. (2019). Community ecology as a framework for human microbiome research. Nat. Med. 25, 884-889. doi: 10.1038/s41591-0190464-9

Goethel, A., Turpin, W., Rouquier, S., Zanello, G., Robertson, S. J., Streutker, C. J., et al. (2019). Nod2 influences microbial resilience and susceptibility to colitis following antibiotic exposure. Mucosal Immunol. 12, 720-732. doi: 10.1038/ s41385-018-0128-y

Goodrich, J. K., Waters, J. L., Poole, A. C., Sutter, J. L., Koren, O., Blekhman, R., et al. (2014). Human genetics shape the gut microbiome. Cell 159, 789-799. doi: 10.1016/j.cell.2014.09.053

Greenhalgh, K., Meyer, K. M., Aagaard, K. M., and Wilmes, P. (2016). The human gut microbiome in health: establishment and resilience of microbiota over a lifetime. Environ. Microbiol. 18, 2103-2116. doi: 10.1111/1462-2920.13318

Gupta, V. K., Paul, S., and Dutta, C. (2017). Geography, ethnicity or subsistencespecific variations in human microbiome composition and diversity. Front. Microbiol. 8:1162. doi: 10.3389/fmicb.2017.01162

He, Y., Wu, W., Zheng, H. M., Li, P., McDonald, D., Sheng, H. F., et al. (2018). Regional variation limits applications of healthy gut microbiome reference ranges and disease models. Nat. Med. 24, 1532-1535. doi: 10.1038/s41591-0180164- $\mathrm{x}$

Ho, H. E., and Bunyavanich, S. (2018). Role of the microbiome in food allergy. Curr. Allergy Asthma Rep. 18:27. doi: 10.1007/s11882-018-0780-z

Holscher, H. D. (2017). Dietary fiber and prebiotics and the gastrointestinal microbiota. Gut Microbes 8, 172-184. doi: 10.1080/19490976.2017.1290756

Hryckowian, A. J., Van Treuren, W., Smits, S. A., Davis, N. M., Gardner, J. O., Bouley, D. M., et al. (2018). Microbiota-accessible carbohydrates suppress Clostridium difficile infection in a murine model. Nat. Microbiol. 3, 662-669. doi: 10.1038/s41564-018-0150-6

Hsiao, A., Ahmed, A. M., Subramanian, S., Griffin, N. W., Drewry, L. L., Petri, W. A., et al. (2014). Members of the human gut microbiota involved in recovery from Vibrio cholerae infection. Nature 515, 423-426. doi: 10.1038/nature13738

Imhann, F., Bonder, M. J., Vich Vila, A., Fu, J., Mujagic, Z., Vork, L., et al. (2016). Proton pump inhibitors affect the gut microbiome. Gut 65, 740-748. doi: 10.1136/gutjnl-2015-310376

Ingrisch, J., and Bahn, M. (2018). Towards a comparable quantification of resilience. Trends Ecol. Evol. 33, 251-259. doi: 10.1016/j.tree.2018.01.013

Jackson, M. A., Verdi, S., Maxan, M. E., Shin, C. M., Zierer, J., Bowyer, R. C. E., et al. (2018). Gut microbiota associations with common diseases and prescription medications in a population-based cohort. Nat. Commun. 9:2655. doi: 10.1038/ s41467-018-05184-7

Jandhyala, S. M., Talukdar, R., Subramanyam, C., Vuyyuru, H., Sasikala, M., and Nageshwar Reddy, D. (2015). Role of the normal gut microbiota. World J. Gastroenterol. 21, 8787-8803. doi: 10.3748/wjg.v21.i29.8787

Jernberg, C., Lofmark, S., Edlund, C., and Jansson, J. K. (2007). Long-term ecological impacts of antibiotic administration on the human intestinal microbiota. ISME J. 1, 56-66. doi: 10.1038/ismej.2007.3

Johnson, A. J., Vangay, P., Al-Ghalith, G. A., Hillmann, B. M., Ward, T. L., ShieldsCutler, R. R., et al. (2019). Daily sampling reveals personalized diet-microbiome associations in humans. Cell Host Microbe 25, 789-802.e5. doi: 10.1016/j.chom. 2019.05.005

Kang, X. X., Yan, J., Huang, F., and Yang, L. (2019). On the mechanism of antibiotic resistance and fecal microbiota transplantation. Math. Biosci. Eng. 16, 7057-7084. doi: 10.3934/mbe.2019354

Karczewski, J., Troost, F. J., Konings, I., Dekker, J., Kleerebezem, M., Brummer, R. J., et al. (2010). Regulation of human epithelial tight junction proteins by Lactobacillus plantarum in vivo and protective effects on the epithelial barrier. Am. J. Physiol. Gastrointest. Liver Physiol. 298, G851-G859. doi: 10.1152/ajpgi. 00327.2009

Kelly, J. R., Borre, Y., O’Brien, C., Patterson, E., El Aidy, S., Deane, J., et al. (2016). Transferring the blues: depression-associated gut microbiota induces neurobehavioural changes in the rat. J. Psychiatr. Res. 82, 109-118. doi: 10.1016/ j.jpsychires.2016.07.019

Kim, M. S., Kim, Y., Choi, H., Kim, W., Park, S., Lee, D., et al. (2020). Transfer of a healthy microbiota reduces amyloid and tau pathology in an Alzheimer's disease animal model. Gut 69, 283-294. doi: 10.1136/gutjnl-2018-317431
Lahti, L., Salojarvi, J., Salonen, A., Scheffer, M., and de Vos, W. M. (2014). Tipping elements in the human intestinal ecosystem. Nat. Commun. 5:4344. doi: 10. 1038 /ncomms5344

Lavelle, A., Hoffmann, T. W., Pham, H. P., Langella, P., Guedon, E., and Sokol, H. (2019). Baseline microbiota composition modulates antibiotic-mediated effects on the gut microbiota and host. Microbiome 7:111. doi: 10.1186/s40168-0190725-3

Le Bastard, Q., Al-Ghalith, G. A., Gregoire, M., Chapelet, G., Javaudin, F., Dailly, E., et al. (2018). Systematic review: human gut dysbiosis induced by nonantibiotic prescription medications. Aliment. Pharmacol. Ther. 47, 332-345. doi: 10.1111/apt.14451

Le Roy, T., Llopis, M., Lepage, P., Bruneau, A., Rabot, S., Bevilacqua, C., et al. (2013). Intestinal microbiota determines development of non-alcoholic fatty liver disease in mice. Gut 62, 1787-1794. doi: 10.1136/gutjnl-2012-303816

Lemon, K. P., Armitage, G. C., Relman, D. A., and Fischbach, M. A. (2012). Microbiota-targeted therapies: an ecological perspective. Sci. Transl. Med. 4:137rv135. doi: $10.1126 /$ scitranslmed.3004183

Liu, R., Chen, P., Aihara, K., and Chen, L. (2015). Identifying early-warning signals of critical transitions with strong noise by dynamical network markers. Sci. Rep. 5:17501. doi: 10.1038/srep17501

Llopis, M., Cassard, A. M., Wrzosek, L., Boschat, L., Bruneau, A., Ferrere, G., et al. (2016). Intestinal microbiota contributes to individual susceptibility to alcoholic liver disease. Gut 65, 830-839. doi: 10.1136/gutjnl-2015-310585

Lloyd-Price, J., Abu-Ali, G., and Huttenhower, C. (2016). The healthy human microbiome. Genome Med. 8:51. doi: 10.1186/s13073-016-0307-y

Lourenco, K. S., Suleiman, A. K. A., Pijl, A., van Veen, J. A., Cantarella, H., and Kuramae, E. E. (2018). Resilience of the resident soil microbiome to organic and inorganic amendment disturbances and to temporary bacterial invasion. Microbiome 6:142. doi: 10.1186/s40168-018-0525-1

Lozupone, C. A., Stombaugh, J. I., Gordon, J. I., Jansson, J. K., and Knight, R. (2012). Diversity, stability and resilience of the human gut microbiota. Nature 489, 220-230. doi: 10.1038/nature11550

Ma, Y., You, X., Mai, G., Tokuyasu, T., and Liu, C. (2018). A human gut phage catalog correlates the gut phageome with type 2 diabetes. Microbiome 6:24. doi: $10.1186 / \mathrm{s} 40168-018-0410-y$

Mach, N., and Fuster-Botella, D. (2017). Endurance exercise and gut microbiota: a review. J. Sport Health Sci. 6, 179-197. doi: 10.1016/j.jshs.2016.05.001

MacPherson, C. W., Mathieu, O., Tremblay, J., Champagne, J., Nantel, A., Girard, S. A., et al. (2018). Gut bacterial microbiota and its resistome rapidly recover to basal state levels after short-term amoxicillin-clavulanic acid treatment in healthy adults. Sci. Rep. 8:11192. doi: 10.1038/s41598-018-29229-5

Maier, L., Pruteanu, M., Kuhn, M., Zeller, G., Telzerow, A., Anderson, E. E., et al. (2018). Extensive impact of non-antibiotic drugs on human gut bacteria. Nature 555, 623-628. doi: 10.1038/nature25979

Manichanh, C., Rigottier-Gois, L., Bonnaud, E., Gloux, K., Pelletier, E., Frangeul, L., et al. (2006). Reduced diversity of faecal microbiota in Crohn's disease revealed by a metagenomic approach. Gut 55, 205-211. doi: 10.1136/gut.2005.073817

Mardinoglu, A., Wu, H., Bjornson, E., Zhang, C., Hakkarainen, A., Rasanen, S. M., et al. (2018). An integrated understanding of the rapid metabolic benefits of a carbohydrate-restricted diet on hepatic steatosis in humans. Cell Metab. 27, 559-571.e5. doi: 10.1016/j.cmet.2018.01.005

Martinez, I., Lattimer, J. M., Hubach, K. L., Case, J. A., Yang, J., Weber, C. G., et al. (2013). Gut microbiome composition is linked to whole grain-induced immunological improvements. ISME J. 7, 269-280. doi: 10.1038/ismej.2012.104

Maya-Lucas, O., Murugesan, S., Nirmalkar, K., Alcaraz, L. D., Hoyo-Vadillo, C., Pizano-Zarate, M. L., et al. (2019). The gut microbiome of Mexican children affected by obesity. Anaerobe 55, 11-23. doi: 10.1016/j.anaerobe.2018.10.009

McBurney, M. I., Davis, C., Fraser, C. M., Schneeman, B. O., Huttenhower, C., Verbeke, K., et al. (2019). Establishing what constitutes a healthy human gut microbiome: state of the science, regulatory considerations, and future directions. J. Nutr. 149, 1882-1895. doi: 10.1093/jn/nxz154

Mehta, R. S., Abu-Ali, G. S., Drew, D. A., Lloyd-Price, J., Subramanian, A., Lochhead, P., et al. (2018). Stability of the human faecal microbiome in a cohort of adult men. Nat. Microbiol. 3, 347-355. doi: 10.1038/s41564-017-0096-0

Menees, S., and Chey, W. (2018). The gut microbiome and irritable bowel syndrome. F1000Res. 7:F1000 Faculty Rev-1029. doi: 10.12688/f1000research. 14592.1

Mondot, S., Lepage, P., Seksik, P., Allez, M., Treton, X., Bouhnik, Y., et al. (2016). Structural robustness of the gut mucosal microbiota is associated with Crohn's 
disease remission after surgery. Gut 65, 954-962. doi: 10.1136/gutjnl-2015309184

Natividad, J. M., and Verdu, E. F. (2013). Modulation of intestinal barrier by intestinal microbiota: pathological and therapeutic implications. Pharmacol. Res. 69, 42-51. doi: 10.1016/j.phrs.2012.10.007

Odamaki, T., Kato, K., Sugahara, H., Xiao, J. Z., Abe, F., and Benno, Y. (2016). Effect of probiotic yoghurt on animal-based diet-induced change in gut microbiota: an open, randomised, parallel-group study. Benef. Microbes 7, 473-484. doi: 10.3920/bm2015.0173

Orwin, K. H., and Wardle, D. A. (2004). New indices for quantifying the resistance and resilience of soil biota to exogenous disturbances. Soil Biol. Biochem. 36, 1907-1912. doi: 10.1016/j.soilbio.2004.04.036

O’Sullivan, O., Cronin, O., Clarke, S. F., Murphy, E. F., Molloy, M. G., Shanahan, F., et al. (2015). Exercise and the microbiota. Gut Microbes 6, 131-136. doi: 10.1080/19490976.2015.1011875

Ott, S. J., Musfeldt, M., Wenderoth, D. F., Hampe, J., Brant, O., Folsch, U. R., et al. (2004). Reduction in diversity of the colonic mucosa associated bacterial microflora in patients with active inflammatory bowel disease. Gut 53, 685-693. doi: 10.1136/gut.2003.025403

Palleja, A., Mikkelsen, K. H., Forslund, S. K., Kashani, A., Allin, K. H., Nielsen, T., et al. (2018). Recovery of gut microbiota of healthy adults following antibiotic exposure. Nat. Microbiol. 3, 1255-1265. doi: 10.1038/s41564-018-0257-9

Peng, J., Narasimhan, S., Marchesi, J. R., Benson, A., Wong, F. S., and Wen, L. (2014). Long term effect of gut microbiota transfer on diabetes development. J. Autoimmun. 53, 85-94. doi: 10.1016/j.jaut.2014.03.005

Philips, C. A., Pande, A., Shasthry, S. M., Jamwal, K. D., Khillan, V., Chandel, S. S., et al. (2017). Healthy donor fecal microbiota transplantation in steroidineligible severe alcoholic hepatitis: a pilot study. Clin. Gastroenterol. Hepatol. 15, 600-602. doi: 10.1016/j.cgh.2016.10.029

Qin, J., Li, R., Raes, J., Arumugam, M., Burgdorf, K. S., Manichanh, C., et al. (2010). A human gut microbial gene catalogue established by metagenomic sequencing. Nature 464, 59-65. doi: 10.1038/nature08821

Ramirez-Farias, C., Slezak, K., Fuller, Z., Duncan, A., Holtrop, G., and Louis, P. (2009). Effect of inulin on the human gut microbiota: stimulation of Bifidobacterium adolescentis and Faecalibacterium prausnitzii. Br. J. Nutr. 101, 541-550. doi: 10.1017/s0007114508019880

Rampelli, S., Schnorr, S. L., Consolandi, C., Turroni, S., Severgnini, M., Peano, C., et al. (2015). Metagenome sequencing of the Hadza hunter-gatherer gut microbiota. Curr. Biol. 25, 1682-1693. doi: 10.1016/j.cub.2015.04.055

Raymond, F., Ouameur, A. A., Deraspe, M., Iqbal, N., Gingras, H., Dridi, B., et al. (2016). The initial state of the human gut microbiome determines its reshaping by antibiotics. ISME J. 10, 707-720. doi: 10.1038/ismej.2015.148

Relman, D. A. (2012). The human microbiome: ecosystem resilience and health. Nutr. Rev. 70(Suppl. 1), S2-S9. doi: 10.1111/j.1753-4887.2012.00489.x

Robertson, S. J., Geddes, K., Maisonneuve, C., Streutker, C. J., and Philpott, D. J. (2016). Resilience of the intestinal microbiota following pathogenic bacterial infection is independent of innate immunity mediated by NOD1 or NOD2. Microbes Infect. 18, 460-471. doi: 10.1016/j.micinf.2016.03.014

Rodriguez-Morato, J., Matthan, N. R., Liu, J., de la Torre, R., and Chen, C. O. (2018). Cranberries attenuate animal-based diet-induced changes in microbiota composition and functionality: a randomized crossover controlled feeding trial. J. Nutr. Biochem. 62, 76-86. doi: 10.1016/j.jnutbio.2018.08.019

Routy, B., Le Chatelier, E., Derosa, L., Duong, C. P. M., Alou, M. T., Daillere, R., et al. (2018). Gut microbiome influences efficacy of PD-1-based immunotherapy against epithelial tumors. Science 359, 91-97. doi: 10.1126/ science.aan 3706

Schaubeck, M., Clavel, T., Calasan, J., Lagkouvardos, I., Haange, S. B., Jehmlich, N., et al. (2016). Dysbiotic gut microbiota causes transmissible Crohn's diseaselike ileitis independent of failure in antimicrobial defence. Gut 65, 225-237. doi: 10.1136/gutjnl-2015-309333

Scheffer, M., Bascompte, J., Brock, W. A., Brovkin, V., Carpenter, S. R., Dakos, V., et al. (2009). Early-warning signals for critical transitions. Nature 461, 53-59. doi: $10.1038 /$ nature 08227

Sharma, S., and Tripathi, P. (2018). Gut microbiome and type 2 diabetes: where we are and where to go? J. Nutr. Biochem. 63, 101-108. doi: 10.1016/j.jnutbio.2018. 10.003

Sharon, G., Cruz, N. J., Kang, D. W., Gandal, M. J., Wang, B., Kim, Y. M., et al. (2019). Human gut microbiota from autism spectrum disorder promote behavioral symptoms in mice. Cell 177, 1600-1618. doi: 10.1016/j.cell.2019.05.004

Shaw, L. P., Bassam, H., Barnes, C. P., Walker, A. S., Klein, N., and Balloux, F. (2019). Modelling microbiome recovery after antibiotics using a stability landscape framework. ISME J. 13, 1845-1856. doi: 10.1038/s41396-0190392-1

Sommer, F., Anderson, J. M., Bharti, R., Raes, J., and Rosenstiel, P. (2017). The resilience of the intestinal microbiota influences health and disease. Nat. Rev. Microbiol. 15, 630-638. doi: 10.1038/nrmicro.2017.58

Stewart, C. J., Ajami, N. J., O’Brien, J. L., Hutchinson, D. S., Smith, D. P., Wong, M. C., et al. (2018). Temporal development of the gut microbiome in early childhood from the TEDDY study. Nature 562, 583-588. doi: 10.1038/s41586018-0617-x

Tap, J., Furet, J. P., Bensaada, M., Philippe, C., Roth, H., Rabot, S., et al. (2015). Gut microbiota richness promotes its stability upon increased dietary fibre intake in healthy adults. Environ. Microbiol. 17, 4954-4964. doi: 10.1111/1462-2920. 13006

Taroncher-Oldenburg, G., Jones, S., Blaser, M., Bonneau, R., Christey, P., Clemente, J. C., et al. (2018). Translating microbiome futures. Nat. Biotechnol. 36, 10371042. doi: $10.1038 /$ nbt. 4287

Vrieze, A., Van Nood, E., Holleman, F., Salojarvi, J., Kootte, R. S., Bartelsman, J. F., et al. (2012). Transfer of intestinal microbiota from lean donors increases insulin sensitivity in individuals with metabolic syndrome. Gastroenterology 143:e917. doi: 10.1053/j.gastro.2012.06.031

Walker, A. W., Ince, J., Duncan, S. H., Webster, L. M., Holtrop, G., Ze, X., et al. (2011). Dominant and diet-responsive groups of bacteria within the human colonic microbiota. ISME J. 5, 220-230. doi: 10.1038/ismej.20 10.118

Willing, B. P., Russell, S. L., and Finlay, B. B. (2011). Shifting the balance: antibiotic effects on host-microbiota mutualism. Nat. Rev. Microbiol. 9, 233-243. doi: 10.1038/nrmicro2536

Wu, G. D., Chen, J., Hoffmann, C., Bittinger, K., Chen, Y. Y., Keilbaugh, S. A., et al. (2011). Linking long-term dietary patterns with gut microbial enterotypes. Science 334, 105-108. doi: 10.1126/science.1208344

Ye, F., Ma, M.-H., Camp, H. J. M. O. D., Chatzinotas, A., Li, L., Lv, M.-Q., et al. (2018). Different recovery processes of soil ammonia oxidizers from flooding disturbances. Microb. Ecol. 76, 1041-1052. doi: 10.1007/s00248-0181183-3

Yeung, A. C. Y., and Richardson, J. S. (2018). Expanding resilience comparisons to address management needs: a response to Ingrisch and Bahn. Trends Ecol. Evol. 33, 647-649. doi: 10.1016/j.tree.2018.06.005

Zaura, E., Brandt, B. W., Teixeira de Mattos, M. J., Buijs, M. J., Caspers, M. P., Rashid, M. U., et al. (2015). Same exposure but two radically different responses to antibiotics: resilience of the salivary microbiome versus longterm microbial shifts in feces. mBio 6:e1693-15. doi: 10.1128/mBio.016 93-15

Ze, X., Duncan, S. H., Louis, P., and Flint, H. J. (2012). Ruminococcus bromii is a keystone species for the degradation of resistant starch in the human colon. ISME J. 6, 1535-1543. doi: 10.1038/ismej.2012.4

Zhao, G., Vatanen, T., Droit, L., Park, A., Kostic, A. D., Poon, T. W., et al. (2017). Intestinal virome changes precede autoimmunity in type I diabetes-susceptible children. Proc. Natl. Acad. Sci. U.S.A. 114, E6166-E6175. doi: 10.1073/pnas. 1706359114

Zuo, T., Lu, X. J., Zhang, Y., Cheung, C. P., Lam, S., Zhang, F., et al. (2019). Gut mucosal virome alterations in ulcerative colitis. Gut 68, 1169-1179. doi: 10.1136/gutjnl-2018-318131

Conflict of Interest: SD and SKD are full time employees of Société des Produits Nestlé. JD received consultancy honoraria from MaaT Pharma, LNC, Biofortis and Nestle and reports grants and/or lecture honoraria from Janssen, Sanofi, MaaT Pharma, BMS, Bridor and Danone.

Copyright $\odot 2020$ Dogra, Doré and Damak. This is an open-access article distributed under the terms of the Creative Commons Attribution License (CC BY). The use, distribution or reproduction in other forums is permitted, provided the original author(s) and the copyright owner(s) are credited and that the original publication in this journal is cited, in accordance with accepted academic practice. No use, distribution or reproduction is permitted which does not comply with these terms. 